\title{
Féeries
}

Études sur le conte merveilleux, XVII $-\mathrm{XIX}{ }^{\mathrm{e}}$ siècle

4 | 2007

Le conte, la scène

\section{Théâtres privés et contes de fées dans la seconde moitié du XVIII ${ }^{\mathrm{e}}$ siècle}

Marie-Emmanuelle Plagnol-Diéval

\section{OpenEdition}

Journals

Édition électronique

URL : http://journals.openedition.org/feeries/253

DOI : $10.4000 /$ feeries. 253

ISSN : $1957-7753$

Éditeur

UGA Éditions/Université Grenoble Alpes

\section{Édition imprimée}

Date de publication : 1 octobre 2007

Pagination : $51-70$

ISBN : 978-2-84310-101-4

ISSN : 1766-2842

Référence électronique

Marie-Emmanuelle Plagnol-Diéval, «Théâtres privés et contes de fées dans la seconde moitié du xvıI siècle », Féeries [En ligne], 4 | 2007, mis en ligne le 02 février 2009, consulté le 08 septembre 2020. URL : http://journals.openedition.org/feeries/253 ; DOI : https://doi.org/10.4000/feeries.253

Ce document a été généré automatiquement le 8 septembre 2020

(c) Féeries 


\title{
Théâtres privés et contes de fées dans la seconde moitié du XVIII ${ }^{\mathrm{e}}$ siècle
}

\author{
Marie-Emmanuelle Plagnol-Diéval
}

1 COLLÉ, EN JUILLET 1755, après avoir assisté à la troisième représentation de Zélide de Renout écrit dans son Journal : «Cette petite féerie (car ce n'est pas une comédie) est une froide et plate imitation de L'Oracle, qui a fait bien des petits. Imitatores servum pecus. M. Renout, qui en est l'auteur, est bien pecus ; il n'y a nulle invention, nul intérêt, point de caractère, rien de neuf dans le fond ni dans les détails ; c'est un amas de madrigaux fades et usés. $M^{\text {lle }}$ Gaussin, par ses grâces divines en ces sortes de rôles, a empêché que cette pièce ne tombât honteusement ${ }^{1} »$. Si critique que soit le jugement de Collé, il dresse le cadre d'une réflexion sur la place de la féerie et plus largement du merveilleux au théâtre dans la seconde moitié du xvıIIe siècle. Il existe (ou il survit) une mode de ces petites pièces, qui tournent autour de fées et d'intrigues amoureuses, favorables au jeu des actrices et dont L'Oracle de Poullain de Saint-Foix est emblématique $^{2}$. La question est donc de savoir comment les théâtres privés, c'est-à-dire les théâtres d'éducation, de société et les fêtes privées, adoptent, reflètent ou adaptent cette mode. Différents éléments porteraient à croire que le merveilleux a toute sa place sur les scènes privées. Les théâtres de société ne partagent-ils pas avec ceux de la Foire une même esthétique de liberté ${ }^{3}$ ? De même, pourrait-on penser que les théâtres d'éducation recourent au merveilleux et à l'univers des contes de fées pour instruire agréablement leurs jeunes auditoires, comme les fêtes privées pour l'expression hyperbolique de leur hommage à leurs commanditaires. Pourtant, l'étude de ces trois corpus dément en partie ces hypothèses. Certes, on trouve quelques pièces merveilleuses dans le théâtre d'éducation, les amateurs de théâtre de société jouent de temps à autre des pièces mettant en scène des personnages merveilleux et quelques fêtes sont effectivement bâties sur une "idée » qui reprend des motifs empruntés au conte, mais ces occurrences sont loin d'être majoritaires en cette seconde moitié du siècle. Pourquoi les prétendues parentés entre le merveilleux et les trois manifestations du théâtre privé qui nous intéressent se révèlent-elles inopérantes? Est-ce une 
question de périodisation ou de nature et donc d'incompatibilité ? Comment alors expliquer et analyser l'élément merveilleux présent? Quelle valeur particulière revêt-il et quelles sont ses modalités d'apparition?

\section{Merveilleux et théâtre d'éducation}

2 «Ennemi de la féerie, la berquinade n'offre pas moins une façon de merveilleux avec ce monde protégé, ces scènes familières, très dialoguées, où les défauts des enfants sont souvent considérés comme des affaires d'État", écrit F. Marcoin à propos de la comtesse de Ségur ${ }^{4}$. Un siècle plus tôt, les éléments en place sont les mêmes. La naissance de la littérature de jeunesse et particulièrement le développement du théâtre "à l'usage des jeunes personnes ", selon la formule consacrée par $\mathrm{M}^{\mathrm{me}}$ de Genlis, s'inscrit dans un refus du merveilleux, assimilé à la superstition et aux préjugés. On se souvient que dans Les Veillées du château, $\mathrm{M}^{\mathrm{me}}$ de Clémire surprend ses deux filles plongées dans la lecture des contes de $\mathrm{M}^{\mathrm{me}} \mathrm{d}$ 'Aulnoy, indûment prêtés par la femme de chambre. La mère démontre aux deux fillettes que ce «merveilleux » qui « n'a rien de vrai » les dégoûte après les avoir enchantées ce qui la conduit au défi suivant: « votre ignorance vous laisse croire que les prodiges et le merveilleux n'existent que dans les contes; la nature et les arts offrent des phénomènes non moins surprenants que les prodigieuses aventures du prince Percinet [...] et pour vous le prouver, je m'engage à faire un conte le plus singulier que vous ayez jamais entendu; et cependant tout le merveilleux en sera vrai ?5 $»$. Ce sera le conte scientifique Alphonse et Dalinde ${ }^{6}$. L'opposition au monde merveilleux, traditionnel ou revu par les contes de fées littéraires de la fin du règne de LouisXIV, s'explique par une présence forte du réel dans les théâtres d'éducation. Si quelques pièces nous apparaissent empreintes d'une certaine invraisemblance psychologique, comme guindées par leur souci démonstratif, il faut les replacer dans le contexte contemporain et se souvenir qu'elles apparurent frappées au sceau d'une vérité absolument nouvelle : celle de l'enfance dans tous ses états, dans tous ses âges et dans toutes ses manifestations. Conçues comme des réponses à une question éducative (la jalousie, la coquetterie, la paresse, etc.), agencées dans des recueils qui se présentent presque comme des manuels, les pièces des théâtres d'éducation frappent les contemporains par une réalité dans laquelle ils veulent reconnaître leurs enfants et se retrouver en tant qu'éducateurs. La conséquence est que les adaptations de contes merveilleux ou les pièces comportant des éléments merveilleux sont rares. Ces derniers sont d'ailleurs rationalisés pour rendre la démonstration pédagogique probante et applicable à l'auditoire concerné. Sur l'ensemble $d u$ corpus des théâtres d'éducation ${ }^{7}$, on ne relève aucune pièce "merveilleuse " chez Nougaret ${ }^{8}$, Moissy, Garnier, Berquin ou $\mathrm{M}^{\mathrm{me}}$ Campan. Il faut cependant mentionner les pièces de $\mathrm{M}^{\mathrm{me}}$ de Graffigny, Ziman et Zénise, Phaza ${ }^{9}$ conçues pour les infants de la cour d'Autriche et dont l'influence sur les pièces merveilleuses de $\mathrm{M}^{\mathrm{me}}$ de Genlis ne saurait être niée. Dans Ziman et Zénise, la fée Bienfaisante a élevé en leur cachant leur origine un couple de jeunes paysans et un couple de jeunes nobles, aujourd'hui âgés de 14 ans pour les filles et de 15 pour les garçons. La pièce met en scène, après une série d'épreuves physiques qualifiantes à l'avantage de Mirflot, l'élection des souverains par les ambassadeurs qui reconnaissent évidemment les qualités morales des jeunes nobles tandis que les petits paysans semblent incapables de se corriger. Quant à Phaza, élevée par la fée Singulière, elle se croit un homme, mais abandonnera bien vite ses idées en présence d'Azor, le fils de la fée Clémentine, ainsi 
qu'il convient à toutes ces pseudo-pièces merveilleuses ${ }^{10}$ qui traitent en fait de l'éveil amoureux de jeunes gens soumis à une expérience éducative dont Marivaux avait donné le modèle. Ainsi, l'oracle qui s'accomplit lors du quinzième anniversaire de la jeune fille ne doit rien aux pouvoirs de la fée, comme le rappelle Clémentine : (« Mon art ne peut rien sur les cœurs, il faut les toucher par adresse ", sc. 2 et " Rappelez-vous que [Phaza] ne peut se soustraire aux pouvoirs de la Fée qu'en se jetant aux pieds d'un vainqueur ", sc. 16).

3 Sur les 37 pièces éditées de $\mathrm{M}^{\mathrm{me}}$ de Genlis, seul figure un petit groupement de pièces du début de sa carrière comportant Les Flacons, La Belle et la Bête dont le titre initial est Zémire et Azor ce qui établit son intertextualité avec Marmontel et L'Île heureuse. Cependant, si nous regroupons ainsi les pièces, leur composition ne suit pas un rythme uni et d'autres pièces s'intercalent dans ces années fructueuses. C'est dire qu'il n'existe pas à proprement parler une veine merveilleuse chez $\mathrm{M}^{\text {me }}$ de Genlis mais plutôt un habit merveilleux qui vient varier un même dessein éducatif et une même dramaturgie. Les trois premières pièces écrites sont Agar dans le désert (une pièce religieuse), Les Flacons (une pièce merveilleuse) et La Colombe (une pièce enfantine réaliste). En quinze jours, $\mathrm{M}^{\mathrm{me}}$ de Genlis compose La Belle et la Bête et L'Enfant gâté. La Belle et la Bête est jouée avec succès durant tout l'hiver 1778 . De cette époque date L'île heureuse. $\mathrm{M}^{\mathrm{me}}$ de Genlis et $\mathrm{M}^{\text {me }}$ Potocka tiennent les rôles de mères et de fées. Les représentations durent huit mois jusqu'à l'été. Le tome I de l'édition de 1779 met un semblant d'ordre dans ces inspirations diverses en s'ouvrant sur Agar dans le désert suivi des trois comédies faisant appel au répertoire merveilleux, puis en se refermant sur trois pièces réalistes (Les Dangers du monde qui mettent en scène de jeunes adultes, les deux saynètes enfantines de L'Enfant gâté et de La Curieuse en 3 actes).

Les échos très flatteurs de ces premières pièces (le théâtre d'éducation est la seule œuvre de $\mathrm{M}^{\mathrm{me}}$ de Genlis qui bénéficie d'une critique unanimement louangeuse) montrent que le public ne distingue pas ces pièces merveilleuses des saynètes réalistes. C'est même cette fonction de realia qui frappe par sa nouveauté et son utilité, en même temps qu'elle justifie l'entrée en écriture d'une femme et d'une mère, plus que ne pourrait le faire l'adaptation théâtralisée de contes de fées littéraires, genre dans lequel se sont illustrées d'autres femmes de lettres. Le recours au merveilleux et ce qu'il pouvait avoir de charmant (les mères en fées, les petites filles face aux flacons magiques, la laideur de la bête et sa transformation ${ }^{11}$ ) n'apparaissent pas dans les poèmes des admirateurs de $\mathrm{M}^{\mathrm{me}}$ de Genlis, qu'il s'agisse du chevalier de Chastellux, de Bonnard ou de La Harpe. Tous louent la nature et la raison, comme dans ces vers de Bonnard :

C'est la vertu, c'est le génie,

C'est la nature et la raison

C'est la grâce à l'esprit unie,

Qui de la décente Thalie

Empruntant la séduction,

Dans un style plein d'harmonie,

Hier nous ont donné leçon. ${ }^{12}$

ou de La Harpe :

Que tes tableaux sont vrais dans leur simplicité !

Tu peins pour des enfants, mais la maturité

Et se reconnaît et t'admire ;

[...] Jamais, jamais la vérité

N'exerça sur les cœurs un plus aimable empire. ${ }^{13}$ 

occur in domestic life, are made the vehicles to improve and delight the young mind ${ }^{14}$ ".

7 Deux pièces de $\mathrm{M}^{\text {me }}$ de Genlis font explicitement référence à un conte. Passons rapidement sur la comédie en un acte, Les Lutins de Kernosi [sic] composée durant l'exil, chez $\mathrm{M}$. et $\mathrm{M}^{\mathrm{me}}$ Cohen, en Allemagne, dont la maison possède un théâtre privé, perdue et jamais imprimée ${ }^{15}$, pour nous attarder un peu sur La Belle et la Bête dont $\mathrm{M}^{\mathrm{me}}$ de Genlis tait la source, mais que lecteurs et spectateurs ne peuvent manquer de replacer dans une série: série narrative avec les récits de $\mathrm{M}^{\mathrm{me}}$ de Villeneuve en 1740 et de $\mathrm{M}^{\mathrm{me}}$ Leprince de Beaumont en 1757 (qui partagent un même lectorat avec $\mathrm{M}^{\mathrm{me}}$ de Genlis), série théâtrale avec Amour pour Amour de Nivelle de La Chaussée en 1742 et Zémire et Azor de Marmontel en $1771^{16}$. La reprise apparente d'un conte traditionnel, ici le « $425 \mathrm{C}$ » dans l'index d'Aarne-Thompson avec le motif de l'époux animal ou monstrueux, n'empêche pas $\mathrm{M}^{\mathrm{me}}$ de Genlis de s'éloigner de la tradition merveilleuse, elle-même revue par le conte littéraire. Les trois femmes utilisent le conte dans des perspectives analogues. $\mathrm{M}^{\mathrm{me}}$ de Villeneuve insère son conte dans un récit intitulé Les Contes marins ou La Jeune Américaine. La femme de chambre d'une jeune fille ayant terminé ses études en France et retournant auprès de ses parents français planteurs à Saint-Domingue distrait sa maîtresse pendant la traversée, au cours de laquelle les autres passagers content également des histoires. Le conte, selon Raymonde Robert est «très moral, voire moralisateur et oppose fortement une héroïne douée de toutes les qualités physiques, mais surtout morales à des sœurs également vaniteuses, égoïstes et insensibles ${ }^{17}$ ». $\mathrm{M}^{\text {me }}$ Leprince de Beaumont, qui écrit pour de jeunes anglaises nobles, allège le conte car son propos est moral et linguistique. $\mathrm{M}^{\text {me }}$ de Genlis le simplifie encore en supprimant un certain nombre d'événements et de personnages. L'héroïne est orpheline et fille unique, ce qui annule les événements antérieurs à son arrivée au palais de la Bête (pas d'errance du père, pas de nuit dans le château mystérieux, pas de pacte avec la bête, pas de sœurs jalouses). Les personnages sont réduits à trois : Zirphée et le génie Phanor, qui ne s'appellent jamais la Belle et la Bête (il n'y a aucune description hyperbolique de la laideur ou de la beauté des héros), la suivante Phédime ${ }^{18}$. Le personnage de l'amie, étrangère au conte qui se joue dans la solitude (solitude des rencontres du père et de la Belle avec la Bête, comme du palais merveilleux, illustrée comme on le sait par le film de J. Cocteau tourné au château de Ranray) est proprement genlisien. L'amie-confidente scande les étapes morales de l'héroïne, sert de porte-parole et de contrepoint, comme le veut l'identification entre l'enfant-spectateur et le personnage, inhérente au théâtre d'éducation.

En réalité, qu'il y ait conte ou simplement accessoires issus d'une culture du conte, $\mathrm{M}^{\mathrm{me}}$ de Genlis rationalise ces éléments, pour construire ses pièces merveilleuses de manière analogue aux autres en récupérant la notion d'épreuve, le schématisme des caractères et la figure du héros. Ainsi les trois pièces féeriques du théâtre d'éducation ont recours au personnel du conte (génie dans La Belle et la Bête, fées dans Les Flacons et L'île heureuse) et soulignent leur place dans une hiérarchie du pouvoir et de la morale :

Phanor: Barbare fée! jouis de l'excès de ma douleur; ton pouvoir, supérieur au mien me condamna jadis à supporter la vie sous cette forme affreuse, et je ne puis reprendre mes premiers traits qu'en parvenant à me faire aimer, qu'en touchant avec cette figure épouvantable, une âme insensible jusqu'alors (La Belle et la Bête, sc. 2). 


\section{pouvoir pour bâtir une épreuve à caractère pédagogique :}

Je leur ai fait croire que je les avais rendues hideuses, et par mon art je leur ai fasciné les yeux de manière qu'en se regardant dans un miroir, et en se voyant l'une l'autre, elles se trouvent affreuses (sc. 1).

Mais on remarquera que la suite échappe au registre merveilleux. Certes, les enfants ont les yeux "fascinés", mais la relativité des canons de beauté est bien la leçon qu'entend prodiguer la pièce et on pourrait imputer à la seule subjectivité le jugement dépréciatif qu'elles portent sur elles-mêmes, ce que conforte la réaction de la mère :

Les pauvres petites n'osent approcher, elles craignent que leur figure ne me fasse horreur. [...] À part. Feignons de ne pas les connaître. (Haut) Mes enfants ne viennent point, je vais aller les chercher... (sc. 2).

ou de leur entourage, conditionné par la fée, comme pourrait le faire une mère avec sa domesticité pour prévenir dans un cadre réel la coquetterie de sa fille...

J'ai donné le mot à tout ce qui les entoure; on leur a répété à chaque instant les premiers jours qu'elles étaient laides à faire peur (sc. 1)

Le choix personnel, certes présent dans le conte, passe au premier plan dans la pièce car il donne à l'épreuve sa valeur éducative. La fée, qui pourrait les douer de «toutes les vertus et toutes les grâces de l'esprit en partage », les fait choisir entre deux flacons, l'un « couleur de rose » qui fera disparaître leur laideur, l'autre blanc qui les rendra parfaites:

Mais il faut choisir, je ne puis vous accorder ces deux dons réunis, mon pouvoir ne va pas jusque-là. (sc. 3 )

13 Évidemment, les flacons n'ont aucun pouvoir, ils ne sont que la métaphore quasi lexicalisée d'un choix de valeurs (l'apparence ou l'intériorité) laissé au libre-arbitre des fillettes sans que la mère intervienne :

\section{La fée à Mélinde}

En vérité, un moment de plus, et vous gâtiez mon épreuve. (sc. 3)

La saynète fait ainsi coïncider deux discours, l'un éducatif que l'on retrouve dans les autres pièces et l'autre issu du conte merveilleux littéraire avec ses topoï : les flacons, les couleurs symboliques, la prétendue laideur des fillettes qui évoquent les créatures disgraciées de $\mathrm{M}^{\mathrm{me}} \mathrm{d}$ 'Aulnoy (rousses et bossues). Le dénouement fait d'ailleurs disparaître l'argument merveilleux au profit de l'envoi éducatif. Ayant retrouvé leur beauté alors qu'elles ont fait le bon choix, les fillettes se demandent avec effroi si elles se sont trompées :

$$
16 \text { Cénie }
$$

Mais par quel prodige le flacon blanc...

17 Ce à quoi la fée répond par un discours qui nie le merveilleux en exaltant l'épreuve pédagogique et la preuve morale qu'elle vient d'administrer :

Après l'action que vous venez de faire, vous n'êtes plus des enfants. Je ne dois plus vous tromper ; tout ce qui vous est arrivé n'était qu'une épreuve. [...] En choisissant le flacon blanc, c'était presque prouver que vous n'en n'aviez pas besoin (sc. 5)

Peut-on dire plus clairement au public enfantin de la pièce et aux éducateurs que le merveilleux n'existe pas?

19

On assiste donc à un effacement du fonctionnement merveilleux au profit du pédagogique, ce que met en évidence L'Île heureuse avec ses deux princesses et leurs 
deux fées gouvernantes Bienfaisante et Lumineuse, plus proche des utopies éducatives que du conte. Dans L'île heureuse, le vote obéit à un déroulement que les entretiens des fées et les conversations des deux princesses ont préparé dans l'esprit du spectateur. La teneur des fausses péripéties (la suivante d'une princesse se méprend sur le résultat du vote) et du coup de théâtre (Clarinde décide de partager son trône avec Rosalide) ne font que renforcer la leçon morale. Dans La Belle et la Bête, c'est la découverte de la charité de Phanor et surtout de la discrétion de sa bienfaisance (deux éléments fondamentaux de l'univers genlisien) qui opèrent peu à peu la conversion de Zirphée : l'argument du conte se réduit à ce choix récurrent entre beauté physique et beauté morale. Il s'agit toujours de valoriser les qualités de cœur et de modestie, de fustiger la vanité et la coquetterie, comme dans La Bonne Mère et Le Voyageur destinés aux jeunes adultes, ou dans La Lingère destinée aux enfants d'humble extraction. Le monde réel et social reste intensément présent, en dépit de l'habillage merveilleux : les fées sont des substituts des mères, elles-mêmes étant des figurations de $\mathrm{M}^{\mathrm{me}}$ de Genlis (ce que perçoivent fort bien les contemporains), les princesses de L'T̂le heureuse sont plurilingues (I, 8) comme le jeune homme modèle du Voyageur (II, 2). Le monde est bien celui des spectateurs et il n'est pas question de le fuir :

Quand vous connaîtrez le monde, vous saurez, mes enfants, comme on doit compter sur les louanges. [...] Il faut le connaître, s'en défier ; ne le point haïr parce qu'il faut y vivre ; et s'en faire estimer parce qu'il nous juge (Les Flacons, sc. 2)

Le dénouement des pièces merveilleuses insiste d'ailleurs sur les valeurs morales par le contenu des dernières répliques et par un système d'énonciation où le personnage se tourne vers le public afin de délivrer la leçon morale :

\section{Phédime}

Soyez toujours heureux, et tous mes vœux seront remplis. (Elle se tourne vers le public). Cœurs sensibles et vertueux, ne vous plaignez jamais du sort; et que cet exemple vous apprenne que la bienfaisance et la bonté sont le seul moyen de plaire et les seuls droits pour être aimé (La Belle et La Bête, II, 5 et d. ${ }^{19}$ )

\section{Mélinde}

N'oubliez jamais, mes enfants, que dans tous les événements de la vie la résolution la plus honnête et la plus vertueuse est toujours la plus sûre et la meilleure (Les Flacons, sc. 5 et d.)

\section{Lumineuse}

Jouissez à jamais, mes chers enfants, du bonheur dont vous êtes si dignes, et n'oubliez point que les plus grands talents et les qualités les plus brillantes ne sont que des dons inutiles ou dangereux, sans la modestie, la bienfaisance et la bonté. (L'T̂le Heureuse, sc. 7 et d.)

\section{Merveilleux et théâtre de société}

Une même déception pourrait se faire jour si l'on examine le répertoire des pièces jouées en société, qu'il s'agisse des pièces créées pour les scènes privées ou de celles que les amateurs importent des théatres officiels. Les adaptations de contes se créent en dehors des théâtres de société et, d'après les témoignages consultés et les anthologies proposées aux amateurs, sont rarement importées. Une recherche par titre à partir du catalogue de Brenner ${ }^{20}$ et des sites ${ }^{21}$ révèlent que les contes traditionnels, révélés au public par Perrault et ses successeurs, font l'objet d'adaptations sous forme de pantomimes ou de féeries, - genres moins pratiqués par les sociétés - pour devenir 
dans la seconde moitié du siècle, à côté de la Foire ${ }^{22}$, le répertoire des troupes d'enfants ou de certains théâtres des boulevards ${ }^{23}$, si bien que la part des théâtres de société est relativement faible. Ainsi, Fuzelier, Lesage et d'Orneval donnent à la Foire SaintGermain un Arlequin roi des ogres ou Les Bottes de sept lieues créé en $1720^{24}$, Beaumarchais reprend le motif des Bottes de sept lieues pour une parade à Étiolles en $1760^{25}$, JeanJulien-Constantin Renout représente un Petit Poucet en 1771-1772 à L'Ambigu Comique et Carmontelle en donne un au tome III des Amusements de société publiés en 1768-1769. Mension fait jouer un Petit Chaperon rouge à L'Ambigu Comique en 1774. Cendrillon inspire à Anseaume un opéra-comique créé le 20 février 1759 à la Foire Saint-Germain, puis Maillé de Marencourt en 1785 au Théâtre de Séraphin, avant de connaître une longue postérité comme en témoigne entre autres un recueil factice intitulé Théâtre de Cendrillon de la bibliothèque des Arts du Spectacle. La Barbe bleue donne successivement lieu à une pantomime d'Adrien-Josephin de Valois d'Orville en 1746 à la Foire SaintLaurent ${ }^{26}$, une tragédie amphigourique de Delautel en un acte donnée au Théâtre de Nicolet en $1766^{27}$, une pantomime donnée à l'Ambigu Comique en 1779 , une pièce donnée par le Théâtre des élèves de l'Opéra en 1780, une tragédie burlesque de François-Martin Poultier d'Elmotte donnée au Théâtre de Lunéville en 1782, auxquelles on pourrait ajouter deux pièces jouées sur des théâtres d'ombres dans les années 1795-1799 et bien sûr la pièce de Sedaine. Nougaret fait jouer Le Chat botté en 1770 à L'Ambigu Comique et le texte est imprimé chez P.-R.-C. Ballard en $1772^{28}$. On note une Belle au bois dormant d'Arnould, créée à L'Ambigu Comique en 1770 et une pantomime donnée à Lyon en $1783^{29}$. Les Souhaits, après la pièce de Regnard en 1700, essaiment sur différentes scènes avec une pièce donnée au collège des Oratoriens de Niort en 1734, un spectacle privé en 1741 à la campagne de $\mathrm{M}^{\text {lle }} \mathrm{de}{ }^{* * *}$, contenant très peu d'éléments merveilleux (publié en 1742 chez J. Barois fils), enfin Le Bûcheron ou Les Trois Souhaits de Jean-François Guichard (musique de Philidor) qui se joue en Belgique et en France de 1763 à $1799^{30}$.

Par ailleurs, les fées et les sorcières, pour notre période ${ }^{31}$, inspirent le Théâtre des grands danseurs (Le Bouquet de la fée Bienfaisante et Les Amours de la fée Carabosse ou Le Bouquet enchanté sont créés en 1779). Ce motif du bouquet enchanté inspire au moins 3 pièces: un opéra-comique anonyme de 1752 (Le Bouquet enchanté ou Les Fleurs indiscrètes ${ }^{32}$ ), Le Bouquet enchanté joué au théâtre de Nicolet en 1769 et au théâtre des Grands Danseurs du Roi à Paris en 1770 (anonyme), mais également une comédie en prose de Carmontelle, Le Bouquet enchanté, représentée en société en $1772^{33}$.

Carmontelle écrit également une pièce satirique sous le mode allégorique, que l'on peut lire comme une variation sur le motif des souhaits, intitulée Les Métamorphoses extravagantes, commencée pendant la campagne de Courtrai de 1744, au cheminement révélateur. Il commence par donner la pièce à des particuliers chargés de la remettre à des amis à Paris. Le texte est perdu. Carmontelle soumet ensuite son texte à Rosely qui lui conseille de le retravailler. La pièce est lue et reçue par les comédiens l'année suivante, mais devant les délais, Carmontelle la propose aux Italiens qui la refusent. Il la fait alors éditer ${ }^{34}$, «mais peu de gens voulurent acheter une pièce qui n'avait point été jouée » (Avertissement). Elle est représentée en 1752.

Si les adaptations de conte sont rares, les éléments merveilleux sont nombreux, mais, on s'en doute, détournés vers des fins sentimentales ou parodiques. Comme toujours dès qu'il s'agit des théâtres de société, notre recensement ne peut prétendre à l'exhaustivité. Il s'est principalement attaché aux scènes situées dans la mouvance 
orléaniste, fréquentées par $\mathrm{M}^{\text {mes }}$ de Genlis et de Montesson, illustrées par Collé, Carmontelle et Laujon ainsi qu'aux anthologies théatrales proposées aux amateurs en panne d'inspiration pour leurs divertissements. Tout d'abord quelle y est la part de merveilleux ? Suivant en cela les théâtres privilégiés et non-officiels, on note sinon une baisse, du moins une minorité de pièces merveilleuses. Peu d'exemples donc, mais d'autant plus remarquables et dont il convient d'étudier les particularités. On se permettra de reprendre la distinction établie dans notre ouvrage ${ }^{35}$ entre les pièces composées tout exprès pour les scènes des particuliers, celles qui sont importées des autres théâtres et enfin les propositions éditoriales.

production que l'on connaît, quatre titres montrent en quel sens est travaillé l'élément merveilleux sur les scènes privées. Outre sa comédie du Chapeau magique tirée de $L a$ Queue de vérité de Fuzelier, Lesage et d'Orneval, et proche du Chapeau de Fortunatus de Fournier ${ }^{36}$ qui réduit l'élément merveilleux à une tromperie destinée au niais, Collé est l'auteur d'une adaptation du roman de Crébillon, L'Écumoire ou Tanzaï et Néadarné, intitulé tragi-comédie ou drame, en un acte et en vers publié en 1768. La pièce illustre le goût de certains théâtres de société pour les parodies à connotation sexuelle de la tragédie orientale ${ }^{37}$. Très proche du roman de Crébillon, elle met en scène outre les héros éponymes, et Saugrenutio, le génie Jonquille et la fée Moustache. La scène d'exposition entremêle un récit de sortilège à une évocation scabreuse des impossibilités amoureuses (« La nuit de mon hymen, une Fée en courroux / Attacha une écumoire à mon illustre époux ", sc. 1), les démêlés de Tanzaï avec l'écumoire et le grand-prêtre rappellent les interdits liés à l'oracle (sc. 2), Jonquille apparaît et disparaît sur un nuage. Le charme qu'il faut rompre sert de prétexte à des considérations érotiques :

30 Saugrenutio

Je ne suis point Génie... et si, sans me flatter,

Mon pouvoir suffira pour vous désenchanter,

Je détruirai le charme, en dépit de l'oracle (sc. 6).

La bégueule héroïque, à Jonquille prêtée

Aurait dû dans son île être désenchantée (sc. 7).

31

Tanzaï

À ces mots qu'à l'instant j'interromps

Par des discours plus vifs et des effets plus prompts

J'entreprends et je crains, mais un coup de tonnerre

Par le seul mouvement d'une peur salutaire

M'a fait rompre le charme. (sc. 9)

et ludiques :

33

\section{Moustache}

Jonquille en a, je vois, passé sa fantaisie ;

Tanzaï n'en sait rien ; c'est un coup de génie !

Mais en si peu de temps, comment? ... Je vois comment;

C'est qu'ils étaient tous deux pressés du dénouement. (sc. 9)

34 L'Île sonnante est composée à la demande du duc d'Orléans pour mettre en valeur les talents lyriques de $\mathrm{M}^{\mathrm{me}}$ de Montesson et Collé en fait une parodie de la comédie à ariettes. La distribution oppose les habitants de l'île sonnante (le sultan Vivatché, l'eunuque Piano, la favorite Mélophanie, le magicien Presto, seul être merveilleux avec 
un esprit infernal) aux naufragés habitués à s'exprimer en prose ou en vaudevilles. De nouveau, les motifs merveilleux sont mis au service de variations ludiques. Zerbin, devant les «prodiges" de l'île, se plaint de «la fantaisie aussi de son maître d'aller consulter cette vieille fée sur les succès de [ses] amours » (I, sc. 2) et de l'obscurité de l'oracle. Les chassés-croisés des couples sont rehaussés par la présence de Presto « le magicien du roi» (I, sc. 4), par les interdits de la fée (La fée ne "vous a permis de m'accompagner que sur la promesse que vous lui avez faite de ne me parler jamais de votre amour») et par les projets de vengeance de Presto ("À leur dépens aussi je prétends m'égayer / Par quelque tout de ma folle magie", I, sc.5). L'acte III est consacré au désenchantement de Zerbin et d'Henriette par l'esprit infernal qui arrive en solfiant, muni d'un grimoire et d'une baguette magique (sc. 3) :

Je sème autour de vous le feu de tous côtés

Ce feu magique renferme (Il fait paraître du feu autour du plateau tournant)

La vertu des secrets aux Enfers inventés (III, sc. 7).

En dépit de ces jeux de scène, la pièce créée à Villers Cotterêts en août $1767^{38}$ est un échec dont Collé détaille les raisons dans son Journal : les paroles, les acteurs, le goût de la société amie des madrigaux et ennemie de toute gaieté, l'absence de feuilles distribuées dans la salle pour faire comprendre les paroles exprimées par la musique, l'oubli « où sont tombés les refrains des vieux vaudevilles ${ }^{39}$ ». Si Laujon réserve ses parodies pour les Italiens (Armide ${ }^{40}$, La Fille, la Veuve et la Femme, Zéphire et Fleurette) quoique le genre s'épanouisse sur les scènes privées avec Collé et Carmontelle, on relève une exception avec Matroco, représenté à Chantilly en 1777 et aux Italiens en 1778, un drame burlesque qui mêle la parodie de la tragédie à celle des poèmes et des romans de chevalerie :

Les cérémonies et sacrifices magiques, les métamorphoses, les désenchantements, les délivrances des chevaliers, leurs combats avec les géants et les nains, les attaques et brisements de tours ${ }^{41}$.

Projet souligné par la liste des personnages qui rassemble entre autres Matroco un enchanteur malfaisant, Furion le chef des géants, les princesses Gloriane et Vaporosine, Emphasis et Fleurdiris les amoureux, une fée, des géants et des nains... Carmontelle, de son côté, fournit une version remaniée du Petit Poucet. Les enfants ne sont que quatre, l'ogre n'a pas de filles (ce qui supprime l'épisode sanglant de leur mort) et un seigneur bienveillant règle le dénouement : «Il veut se charger de les faire élever, et puis après de leur faire apprendre un métier à chacun ».

Certains théâtres de société servent de scènes d'essai comme chez la duchesse de Villeroy, rue de l'Université, où l'on monte, à grand renfort de machines et de décors, le "ballet figuré » $L a$ Tour enchantée de Nicolas-René Joliveau ${ }^{42}$ sur une musique de Dauvergne et des ballets de Laval, qui sera repris en juin 1770 à Versailles, ainsi que $L a$ Sorcière par hasard, un opéra-comique de Nicolas-Étienne Framery créé en 1768 avant une première à la Comédie-Italienne en $1783^{43}$.

Pourtant la féerie, sinon la pièce merveilleuse, attire plutôt la méfiance des auteurs appointés du théâtre de société, écartelés entre l'obligation de satisfaire l'attente des commanditaires, des acteurs-amateurs, des spectateurs et le désir croissant de sortir de ce statut d'amuseur pour gagner celui de véritable auteur. Sans doute, doit-on ainsi comprendre la critique de Collé à l'égard des féeries et les distinctions génériques qu'il avance en faveur de la véritable comédie, celle-là même qu'il s'efforce d'écrire à partir de La Partie de chasse et de ses adaptations des Illustres Françaises. Mais inversement, le 
merveilleux ajoute au plaisir du travestissement inhérent au théâtre amateur. Ainsi, les personnages de fées et d'enchanteurs ravissent les acteurs. On ajoute des rôles merveilleux à des textes qui n'en comportent pas à l'origine, suivant cette pratique courante des scènes privées qui veulent avant tout satisfaire les acteurs et les assistants. $\mathrm{M}^{\mathrm{me}}$ de Genlis détaille comment elle compose pour la petite société du Vaudreuil une pièce, aujourd'hui perdue, tirée de la légende locale de la Montagne des deux amants, chaînon d'une longue série narrative et théâtrale ${ }^{44}$. Hormis la notion d'épreuve imposée par le père, rien dans la légende plus ou moins toponymique ne suggère un élément merveilleux comme le souligne $\mathrm{M}^{\mathrm{me}}$ de Genlis : "Telle est la tradition, qui a l'air d'une allégorie, car, en effet, l'amour promet tout, entreprend tout, et après avoir tout obtenu, il expire! ». Après avoir fait en groupe l'ascension de la montagne, fait construire un "petit théâtre charmant dans l'orangerie » et écrit la pièce en deux jours, $\mathrm{M}^{\mathrm{me}} \mathrm{de}$ Genlis distribue les rôles et se réserve celui d'un enchanteur, absent de la trame originelle, «qui avait 200 ans et que je supposais établi sur la montagne inaccessible, où il devait rester jusqu'à l'arrivée de deux amants parfaits, il les attendait depuis plus d'un siècle et demi». Et d'ajouter: "J'étais enchantée de mon rôle, parce que j'avais une perruque et une barbe blanche ». Le travestissement avec changement de sexe et d'âge, le costume, l'accessoire constituent autant de signes de disconvenance qui font partie du jeu social et dramatique, en même temps que l'ajout merveilleux s'autodétruit puisque «la perfection de leur amour mutuel désenchantait le vieux solitaire de la montagne ${ }^{45}$ ». Tout ici est faux : le rôle inventé comme le dénouement de la pièce qui ménage une fin heureuse contraire à la tradition. Le merveilleux n'est qu'un prétexte au contre-emploi et au déploiement de costumes. De Zénéide de Cahusac, jouée au château de Grisolles parmi d'autres pièces, $\mathrm{M}^{\mathrm{me}}$ de Genlis retient que sa belle-sœur joue le rôle-titre tandis qu'elle tient le rôle masculin d'olinde "qu'une femme peut jouer parce qu'il est habillé d'un long domino fermé ${ }^{46}$ ». Et lorsqu'on croise les témoignages de Collé et de $\mathrm{M}^{\text {me }}$ de Genlis à propos de l'unique représentation de L'̂̂le sonnante, cette dernière ne mentionne que la splendeur de son costume : «J'y jouais une sultane et j'ouvrais la scène par une longue ariette que je chantais en m'accompagnant de la harpe. Monsigny avait fait l'ariette et le rôle pour moi. J'avais un habit simple, chargé d'or et de pierreries; et quand on leva la toile, je fus applaudie à trois reprises et on me redemanda trois fois mon ariette ${ }^{47}$ ”.

Peu présent dans les pièces composées spécialement pour les théâtres de société, utilisé de manière ludique, érotique ou parodique, le merveilleux séduit cependant quelques commanditaires et acteurs amateurs que n'effraient pas des mises en scène fastueuses. Voyons ce qu'il en est dans le répertoire importé à travers quelques théâtres de société et quelques cercles de sociabilité. Comme influencés par Marivaux, ces répertoires se caractérisent par « l'atmosphère de la féerie, plus que l'action de la féerie ${ }^{48}$. Ainsi, les Mémoires de $\mathrm{M}^{\mathrm{me}}$ de Genlis, qui nous renseignent sur les pièces jouées dans les sociétés qu'elle fréquente, citent peu de pièces que l'on pourrait qualifier de merveilleuses, montrant bien que la mode est plutôt en ces débuts du règne de Louis XVI aux bergeries et aux noces

villageoises d'un pseudo-réalisme campagnard. Comme tant d'autres, elle joue Le Devin $d u$ village (également recommandé par Paulmy d'Argenson ${ }^{49}$ ) dont le rôle-titre doit plus à la connaissance des cœurs de Colin et de Colette qu'à l'arsenal merveilleux traditionnel :

J'ai tout su de Colin, et ces pauvres enfants

Admirent tous les deux la science profonde 
Qui me fait deviner tout ce qu'ils m'ont appris (sc. 3).

[...] Je vous ai délivré d'un cruel maléfice

Vous vous aimez encore malgré les envieux (sc. 7). retrouver chez d'autres grands seigneurs et dans les anthologies contemporaines, on joue Zénéide de Cahusac. La tirade de la fée relatant la naissance de l'héroïne dans l'exposition présente un condensé des motifs merveilleux traditionnels (les pouvoirs des fées et leur rivalité, la scène des dons, l'oracle menaçant), comme autant de clins d'œil au public, pour ensuite céder à une initiation amoureuse entre jeunes gens et à une réflexion aussi légère sur l'amour et le monde que toutes ces autres pièces qui empruntent au monde merveilleux. On joue également pour l'ouverture du théâtre de la rue de la Roquette, les 18, 19 et 20 novembre 1754 pour la sainte Élisabeth, prénom de $\mathrm{M}^{\text {lle }}$ Le Duc, maîtresse du Comte, trois spectacles différents, dont La Coupe enchantée de La Fontaine sous le nom de Champeslé ${ }^{50}$, au croisement du fabliau, du conte et du poème, qui traite du merveilleux sur un mode oblique caractéristique des scènes privées $^{51}$.

Dans les anthologies proposées aux amateurs de théâtre, les pièces d'inspiration merveilleuse sont rares. Aucune pièce dans les comédies du Théâtre Français en 5, 3 et 2 actes chez Paulmy, mais dans celles en un acte La Coupe enchantée de La Fontaine, L'Oracle de Poullain de Saint-Foix et Zénéide de Cahusac. Du côté du Théâtre Italien, Arlequin poli par l'amour, dont on connaît le lien avec le conte de $\mathrm{M}^{\mathrm{me}}$ Durand ${ }^{52}$, apparaît au détour d'une note très significative : «Si dans la société, il se trouvait quelqu'un qui se sentît le genre de talent à remplir le rôle d'Arlequin, on pourrait ajouter à ces pièces celles qui suivent» (p.236), dont La Sylphide de Biancolelli et Romagnesi qui, autour d'Éraste et Arlequin, met en scène une sylphie et une gnomide entourées de sylphes et de sylphides chantant et dansant. Pour les "actes d'opéra ", des passages de La Sibylle (paroles de Moncrif, musique de Dauvergne), Les Génies du feu de L'Empire de l'Amour (paroles de Moncrif, musique de Brassac), pour les pièces à ariettes Le Sorcier de Poinsinet sur une musique de Philidor en dépit d'un rôle-titre difficile selon Paulmy.

Par ailleurs, on sait que les théâtres de société raffolent des fêtes et organisent des spectacles composites dans lesquels le théâtre, la danse, la musique, les arts lyriques et parfois la pyrotechnie se mêlent pour célébrer le commanditaire, l'hôte, un invité de marque ou une occasion particulière telle qu'une fête de saint patron, un anniversaire, une convalescence ou l'inauguration d'un lieu. Ces fastes, liés à un certain type de sociabilité, qui renvoient au groupe une image volontairement construite de lui-même s'organisent autour d'une idée, le plus souvent empruntée aux fêtes princières ou aux manifestations organisées par les villes lors du passage d'un personnage célèbre. L'argument de ces fêtes privées est rarement issu du domaine merveilleux : une fête champêtre, une guinguette ou une reconstitution historique plaisante sont souvent préférées pour d'évidentes raisons d'adéquation avec le décor naturel du château ou de son parc. Mais les auteurs-organisateurs reprennent parfois des motifs empruntés au conte comme Laujon dans sa "Fête de jardin dans l'île d'Andrésil » qui met en scène dom Jugement, dame Mémoire, demoiselle Imagination et la fée Bienfaisante ou dans sa "Fête villageoise donnée dans un hameau pour la réception de mademoiselle à Chantilly » le 3 juin 1777, qui promène la jeune fille dans tous les lieux du domaine avec un divertissement et un arrangement théâtral en accord avec chaque lieu, dont une grotte habitée par une sorcière qui évoque plaisamment sa "race de sorcières, de bohémiennes, de diseuses de bonne aventure ", «la réunion de toute [sa] sorcière de 
société » et Nostradamus ${ }^{53}$ (sc. 3). Le motif de la prédiction ou du portrait louangeur se prête à une variante surnaturelle, invoquée par un trait d'esprit comme dans cette fête de société également de Laujon, intitulée La Diseuse de bonne aventure :

Vous êtes étonnées, Mesdames, qu'étant si petite j'en sache si long? Mais la fameuse fée Ninette était une fois plus petite que moi et elle vous lisait tout ce qui se passe dans une tête... au point qu'elle aurait compté toutes les idées folles et légères de nos papillons à la mode $\mathrm{e}^{54}$.

La description des fêtes données à S.A.S Madame la comtesse de Brionne à Limours ${ }^{55}$ rend bien compte de la façon dont quantitativement et qualitativement les éléments merveilleux peuvent trouver place dans un agencement festif, lui-même plus ou moins convenu ${ }^{56}$. Sur huit fêtes données à Limours entre 1775 et 1783, comptant entre autres une fête champêtre (un mai à Limours) et une villageoise (une fête des écoles à Limours), deux seulement contiennent des éléments merveilleux avec la représentation du Devin du village lors des festivités du 30 décembre 1778 pour le mariage du prince de Vaudémont et un cortège pour la sainte Élisabeth, patronne de la comtesse de Vaudémont, célébrée le 20 novembre 1779. On y voit un char " traîné par deux hommes vêtus avec des habits de Diables de l'Opéra ", "sur ce char était assise Mélusine, vêtue en Armide supposée enchantée dans le château de Limours depuis nombre de siècles et dont le désenchantement est censé opéré par l'arrivé de la princesse de Vaudémont dans ce château et Mélusine lui en fait des remerciements ». Paulmy évoque une fête donnée pour l'accouchement de la Marquise de ${ }^{* * *}$, entièrement conçue sur le mode féerique (peut-être en raison du motif de réjouissance, proche de la situation initiale du conte : la fête pour la naissance d'un héritier et les vœux qui l'accompagnent) avec une fête autour du roi des génies, sa suite et la fée Carabosse, puis trois actes se déroulant au milieu de meubles enchantés, d'un jardin enchanté et enfin dans la ménagerie des fées.

Ce survol des scènes amateurs et des différents types de spectacles qu'elles peuvent proposer à leur « société » en cette seconde moitié du siècle montre que les contes et le merveilleux en général ne sont pas les principales sources d'inspiration des auteurs, qu'ils soient improvisés ou appointés par un commanditaire. La bergerie, la saynète enfantine, la parodie des grands genres y sont plus à l'honneur que le merveilleux, même indirectement présent ou détourné vers des fins ludiques ou sensuelles. Les pièces ou les fêtes qui lui font ouvertement place méritent donc une étude particulière car les textes, les agencements scéniques et leurs réceptions constituent de précieux indicateurs sur les mentalités. Au-delà, convient-il sans doute, d'ouvrir l'enquête aux pièces qui sont visiblement influencées par le conte. Dans ce qui pourrait apparaître comme un nouveau répertoire, quatre groupes au moins se font jour autour des thèmes du sylphe, de la statue, de la bergère (qui jouent tous trois sur une fausse identité) et enfin du réveil du héros après un sommeil de plusieurs siècles. Le personnage du sylphe si répandu au xvIIIe siècle ${ }^{57}$, illustré au théâtre entre autres par Martin de Choisy dans Le Sylphe ou le mari comme il y en a peu ${ }^{58}$ donne lieu à des variations sur les entreprises de séduction comme le montreraient L'Amant anonyme de $\mathrm{M}^{\mathrm{me}}$ de Genlis ou La Forte Vapeur de Moissy. De même la bergerie et le motif merveilleux de la bergère aimée du prince se rejoignent dans un certain nombre de pièces où l'héroïne, à la suite de mésaventures, est contrainte de dissimuler son identité comme dans deux pièces du marquis de Montalembert, La Bergère de qualité et La Bohémienne supposée de 1786. Le motif de la statue merveilleuse qui s'anime pour n'être bien sûr que la femme aimée et qui donne son titre à la pièce de Laurès $L a$ Fausse Statue ${ }^{59}$ irrigue un certain nombre de pièces de 
société, dont La Statue de Montalembert. Quant au motif traditionnel du héros sortant d'un long sommeil, il entre dans une série ${ }^{60}$, souvent dirigée vers des fins politiques et connaît un succès durable sur les scènes révolutionnaires après avoir illustré les aventures du parlementaire J.-J. Duval d'Esprémesnil lors d'un spectacle donné pour son retour d'exil de l'île Sainte-Marguerite ${ }^{61}$. L'esprit du merveilleux n'est certes pas mort sur les scènes privées. S'il ne donne pas à voir un univers authentiquement codé, il s'interroge sur la part de merveilleux inhérente aux rencontres amoureuses et aux manœuvres de séduction. Il laisse son empreinte dans le monde idéalisé de l'éducation et se tourne vers le passé comme le montre le projet de Collé de réécrire pour la scène officielle L'Esprit follet ou la dame invisible ${ }^{62}$. Nostalgie, jeux de réécriture et auditoire fluctuant témoignent du statut instable du merveilleux en cette fin de siècle, comme le donne à penser le titre ironique de la pièce de Nougaret, Il n'y a plus d'enfants ${ }^{63} . .$.

\section{NOTES}

1. Journal historique ou Mémoires critiques littéraires, nouvelle édition par Honoré Bonhomme, Paris, Firmin Didot, 1868, II, p. 22-23.

2. L'Oracle a été joué le 3 décembre 2005 à la Bibliothèque de Fresnes par la compagnie des SeptSœurs dans une mise en scène de David Mambouch.

3. Paul Ginisty dans La Féerie, Paris, L. Michaud, 1910 (Rééd. Les Introuvables, Plan-de-la-Tour, Éditions d'aujourd'hui, 1982) écrit: «On trouverait, dans le répertoire de la Foire, cent pièces féeriques » (p. 17).

4. «Le style et la schize ", La Comtesse de Ségur, Europe, juin-juillet 2005, p. 71-93, p. 78.

5. Notre édition de référence est l'édition de 1784, Lambert et Baudoin, I, p. 373-375.

6. Voir notre article "Merveilleux ou rationnel : Les Veillées du château de Mme de Genlis ", dans Actes du séminaire de l'UMR LIRE, CNRS-Lyon 2, Le Partage des savoirs XVIII ${ }^{e}-X I X^{e}$ siècles sous la direction de Lise Andriès, Lyon, PU de Lyon, 2003, p. 151-162.

7. $M^{m e}$ de Genlis et le théâtre d'éducation au XVIII siècle, Oxford, Voltaire Foundation, SVEC vol. 350, 1997.

8. On notera tout au plus dans le Théâtre à l'usage des collèges, des écoles royales et des pensions particulières, Paris 1789, de Pierre-Jean-Baptiste Nougaret une pièce intitulée Gulliver chez les Lilliputiens (t. I) dans laquelle les plus jeunes enfants jouent les habitants de Lilliput.

9. Cuvres posthumes de $M^{m e}$ de Graffigny, Amsterdam, Paris, chez les libraires, 1770.

10. Quelques répliques sont à cet égard significatives. Si l'on assure que Singulière est «transformée en serpent » et qu'elle « rampe actuellement sur la terre » (sc. 1), Clémentine croit à tort que son fils est entré de manière magique dans le château de Phaza (sc. 2). Concernant le théâtre de $\mathrm{M}^{\text {me }}$ de Graffigny, je renvoie bien sûr à l'ensemble des travaux de Charlotte Simonin.

11. Toute chose que les spectateurs de la représentation théâtrale donnée par la DDD Compagnie le 22 juin 2006 ont pu apprécier lors du colloque international « Les Femmes éducatrices au siècle des Lumières ", les 22, 23 et 24 juin 2006, organisé par Isabelle Brouard-Arends et MarieEmmanuelle Plagnol-Diéval à Rennes 2.

12. La Harpe, Correspondance littéraire adressée à S.A.I. Mgrle Grand Duc, aujourd'hui Empereur de Russie, et à M. Le C $C^{\text {te }}$ André Schowalow..., Paris, Migneret, 1801, II, Lettre CV, p. 362. 
13. La Harpe, ouvr. cité, Lettre CIV, II, p. 359-360.

14. "The Theatre of Education, translated from the French of the countess of Genlis, Vol. ii, iii and iv ", The London Magazine or gentleman's monthly Intelligencer, mars, 1781, p. 141.

15. Mme de Genlis attribue ce conte à $\mathrm{M}^{\mathrm{me}} \mathrm{d}$ 'Aulnoy, mais il s'agit plutôt des Lutins du château de Kernosy, de $\mathrm{M}^{\mathrm{me}}$ de Murat, œuvre mentionnée à deux reprises par R. Robert, dans Le Conte de fées littéraire en France de la fin du XVII ${ }^{e}$ siècle à la fin du XVIII ${ }^{e}$ siècle, Presses universitaires de Nancy, 1982, p. 306 et 314. Casimir Becker, l'enfant qu'elle a adopté et converti au catholicisme, y joue à la perfection un rôle de valet auquel il ajoute des traits de son $\mathrm{cru} \mathrm{M}^{\mathrm{me}}$ de Genlis, qui devait à l'origine simplement jouer de la harpe, remplace une jeune actrice trop timide. C'est un succès d'actrice et de femme (le public, dit-elle, lui donne à peine trente ans) et une revanche sur les événements politiques.

16. Sophie Allera et Denis Raynaud ont donné une anthologie commentée de ces textes : La Belle et la Bête, quatre métamorphoses, textes établis et annotés, Publications de l'université de SaintÉtienne, 2002.

17. R. Robert, ouvr. cité, p. 147.

18. Ces noms évoquent un Orient conventionnel.

19. La leçon n'est guère différente chez $\mathrm{M}^{\mathrm{me}}$ Leprince de Beaumont : «Belle, lui dit cette dame qui était une grande fée, venez recevoir la récompense de votre bon choix vous avez préféré la vertu à la beauté et à l'esprit, vous méritez de trouver ces qualités réunies en une même personne».

20. Brenner Clarence D., A bibliographical list of plays in the french language 1700-1789, Berkeley, 1947.

21. CESAR (Calendrier électronique des spectacles d'Ancien Régime) et le site des théâtres de société créé par David Trott et co-animé par Marie-Emmanuelle Plagnol-Diéval et Dominique Quéro

<http://www. chass. utoronto. ca/ trott/societe/societe. htm>.

22. Paul Ginisty (ouvr. cité) indique, concernant la genèse du genre : "C'est au théâtre de la Foire qu'est le vrai berceau de la féerie. Elle va s'affirmer à la Comédie-Italienne, puis, peu à peu retomber dans la farce»(p.47). On peut ne pas partager ce jugement esthétique, qui rend néanmoins compte du cheminement du merveilleux sur les différentes scènes.

23. Voir notre article, "Enfants, troupe d'enfants et répertoires au XVIII ${ }^{\mathrm{e}}$ siècle », communication pour le colloque international "Troupes et jeunesse », organisé par Christiane Page, université d'Artois, Arras, les 22 et 23 mars 2005, Cahiers Robinson, n 18, 2005, p. 9-20.

24. Arlequin fait naufrage dans l'île des ogres qui attendent un roi géant pourvoyeur de toutes les denrées grâce à « ces fameuses bottes dont il est fait mention dans l'histoire des fées » (sc. 10). 25. Les annonces de la parade revendiquent l'hypotexte de Perrault: «C'est z'icy que l'on voit cette fameuse paire de fées, ces fameuses bottes du fameux Petit Poucet, que la fameuse histoire, composée par ce fameux Monsieur Perrault, z'a rendues si fameuses dans tout le fameux univers du monde entier [...]».

26. Ms. BNF, f. 9318

27. Ms. BNF, f. 9248

28. La pièce fait partie d'un spectacle joué par la troupe d'Audinot pour Louis XV et $\mathrm{M}^{\mathrm{me}}$ du Barry à Choisy le 8 avril 1772 comprenant une comédie au titre évocateur de Nougaret Il n'y a plus d'enfants, deux pantomimes (La Guinguette d'Audinot et Le Chat botté), puis une contredanse suggestive, appelée « la fricassée ». Le canevas de la pantomime " est tiré des contes de fées de Perrault » (note sur la page de titre du Recueil de fêtes et spectacles donnés devant Sa Majesté, à Versailles, à Choisy et à Fontainebleau, Paris, Ballard, 1772) et reprend en effet les principaux épisodes du conte : l'héritage, la chasse, les manœuvres du Chat Botté auprès du Seigneur et de sa fille, l'habit donné au fils du meunier, les paysans menacés d'être réduits en chair à pâté, la souris 
sauvée par le Chat Botté qui se transforme en fée et le mariage agrémenté ici d'un divertissement où l'on voit le Chat Botté sortir d'un pâté, puis danser avec un âne.

29. Brenner indique une autre, une comédie féerique, Ms. BNF, n.a.f. 2873, sans indication de représentation, prélude à une immenses postérité au $\mathrm{XIX}^{\mathrm{e}}$ siècle.

30. Brenner signale en outre une comédie en un acte, Ms. BNF, n.a.f. 2994.

31. C'est pourquoi nous ne parlons pas, entre autres des contributions de Caylus à Morville et notamment de sa comédie, Les Âges ou La Fée du Loreau représentée le 20 septembre 1739. Voir à cet égard Le Comte de Caylus, les arts et les lettres, Actes du colloque international, Université d'Anvers (UFSIA) et Voltaire Foundation, Oxford, 26 au 26 mai 2000, études réunies et présentées par Nicholas Cronk et Kris Peeters, Rodopi, Amsterdam New-York, 2004.

32. Ms. BNF, f.fr. 9295.

33. Sans autre indication de publication ou de manuscrit.

34. La Bibliothèque de l'Arsenal possède un exemplaire imprimé (Bruxelles, P.-J. Lemmans, 1748) et un manuscrit Ms. Arsenal 9446.

35. Le Théâtre de société: un autre théâtre?, «Les Dix-huitièmes siècles ", collection dirigée par Raymond Trousson et Antony McKenna, Paris, Honoré Champion, 2003.

36. Publié dans le Théâtre des Boulevards ou Recueil des parades, Mahon, G. Langlois, 1756.

37. Voir notre article, "Séries parodiques dans le théâtre de société ", dans Séries parodiques au siècle des Lumières, Textes réunis par Sylvain Menant et Dominique Quéro, PUPS, 2005, p. 123-149. 38. La pièce est ensuite jouée par les Italiens le 4 janvier 1768 et Collé rapporte le jugement négatif d'un critique : la satire de la pièce à ariettes n'est pas vue et la comédie est considérée comme un amphigouri de société, tout au plus une parodie d'Ernelinde. Elle se maintient ensuite à la Comédie-Italienne, attelée avec Les Moissonneurs de Favart ou Le Peintre amoureux de son modèle avec quelques réfections.

39. Collé, ouvr. cité, t. III, p. 156.

40. La parodie d'Armide disqualifie constamment le pouvoir merveilleux, à la source du conte. Ainsi Hidraot, incapable d'avoir un « rejeton » (comme Alphonse l'impuissant de Collé) se plaint en ces termes :

Mon art fait tout trembler / Mais je n'ai pu peupler / Quoique sorcier (I, sc. 2) / Le même est également incapable de faire venir Renaud et fait patienter Armide : / S'il était ici, ma mie, / Ta baguette et ma magie / M'en auraient sans doute instruit (II, sc. 2) / Le déclassement parodique touche ici tous les éléments merveilleux comme il le fait avec l'univers tragique, en transformant les Démons en nourrices et nourriciers pour endormir Renaud (II, sc. 3), puis en zéphyrs (II, sc. 6) et en traitant Amide de « sorcière " (IV, sc. 3).

41. Laujon, Euvres choisies contenant ses pièces représentées sur nos principaux théâtres, sur ceux de province ou de société, ses fêtes publiques ou particulières; ses chansons et autres opuscules, avec des anecdotes, remarques et notices relatives à ces divers genres, Paris, Patris, 1811, 4 vol., t. III, Avertissement, p. 3.

42. Le texte de Joliveau met en scène une fée (la reine des îles d'Or, une princesse, Renaud, Florestan et Margian un génie malfaisant). À la scène 1, "Le théâtre représente le palais souterrain de Margian. On a exprès affecté de traiter cette décoration dans un genre également lourd et bizarre ». Margian s'adresse aux génies malfaisants pour le seconder avec force démons, serpents, torches et poignards. La scène 2 se situe dans un camp au bord de la mer « au milieu de laquelle représente on voit une tour lumineuse gardée et défendue par des géants et des monstres » et les adversaires se livrent bataille à la scène 5 : Margian est sur un char traîné par des griffons qui jettent des flammes et « la tour enchantée se brise, un nuage enlève la princesse, et la Reine paraît dan un char éclatant ».

43. La pièce est publiée à Paris, chez Houbaut, en 1783. 
44. La légende racontait qu'un jeune homme avait relevé le défi de grimper au sommet de la montagne, il mourut et son amante se jeta de désespoir dans la rivière... Voir H. Coulet, Le Conte de deux amants à la fin du XVII siècle et au début du XIX siècle, Wolfenbütteler Forschungen, Formen innerliterischer Rezeption, 1987, colloque 29 mai-1 ${ }^{\text {er }}$ juin 1985, p. 99-111.

45. Mémoires..., Paris, Ladvocat, 1825, II, p. 72-76.

46. Mémoires... I, p. 273.

47. Mémoires... I, p. 343.

48. Paul Ginisty, ouvr. cité, p. 50.

49. Paulmy A.-R. de Voyer d'Argenson $\mathrm{M}^{\mathrm{is}}$ de, et Contant d'Orville, Mélanges tirés d'une grande bibliothèque (Par A.-R de Voyer d'Argenson, marquis de Paulmy et A.-G. Contant d'Orville), II Manuel des châteaux ou Lettres contenant des conseils pour former une bibliothèque romanesque, pour diriger une comédie de société, et pour diversifier les plaisirs d'un salon;également appelé Manuel des sociétés qui font leur amusement de jouer la comédie ou Catalogue raisonné de toutes les tragédies, comédies des théâtres français et italien (1779-1788) encore appelé Étrennes aux sociétés...

50. J. Cousin, Le Comte de Clermont, sa cour et ses maîtresses. Lettres et documents inédits, publication de M. Jules Cousin, Paris, Académie des bibliophiles, 1867, 2 vol., II, p. 58-61.

51. Devant les prodiges attribués à la coupe, Thibaut s'écrie : «Cela est bouffon!» (sc. 6) et, plus sage que les autres, refuse de tenter l'épreuve (sc. 18).

52. Voir Shirley E. Jones, «A probable source of Marivaux's Arlequin poli par l'amour », French Studies, 1965, p. 385-391 et l'analyse de F. Deloffre et F. Rubellin, Marivaux, Théâtre complet, La Pochothèque, Classiques Garnier, Paris, 2000, notice p. 111-123.

53. Nostradamus intervient à plusieurs reprises, moins comme personnage historique que comme devin merveilleux. Il en est ainsi dans La Relation des réjouissances que le collège de Belzunce et la maison des pensionnaires du même collège ont faites à l'occasion de la naissance de Mgr le duc de Bourgogne.

54. Laujon, t. II, p. 370-371.

55. Je remercie Michèle Crogiez de m'avoir signalé ce document, BNF, Ms. 14441, disponible sur Internet.

56. Ainsi dans l'opuscule des Fêtes données à S.A.S $M^{\text {me }}$ la Duchesse au mois d'août, Forges, 1737, la première fête, le $1^{\text {er }}$ août 1737 suit un déroulement codé d'un défilé champêtre et la seconde, une semaine plus tard, utilise un argument mi-merveilleux, mi-allégorique: «dont l'idée est la défaite de la fée Vaporine, par les charmes de la fée des Plaisirs. Cette pièce [...] commença par l'ouverture des Indes Galantes, suivie d'un monologue de la fée Vaporine. Cette scène moitié récit, moitié en vers burlesques, et en style de vaudevilles, ainsi que tout le reste de ce premier acte, contient les inquiétudes de la fée Vaporine sur sa prochaine destruction, annoncée par un songe d'un goût singulier» (p. 23). "L'acte est terminé par un chœur de farfadets et de lutins, suivi d'un ballet de démons et d'une entrée de furies, tous habillés d'habits d'Opéra, venus exprès de Paris » (p. 24). « Le second acte commence par une marche grave et sérieuse pour annoncer la venue de l'enchanteur Merlin ».

57. Sylphes et sylphides, anthologie établie et présentée par Michel Delon, Paris, Desjonquères, 1999.

58. Montpellier, J.-F. Picot, 1778.

59. Paris, Le Jay, 1771.

60. Poisson est l'auteur d'un Réveil d'Épiménide de Poisson créé en 1735, de même que le président Hénault en 1755 et Carbon de Flins des Oliviers auteur d'un Réveil d'Épiménide à Paris créé le $1^{\text {er janvier }} 1790$ au théâtre de la Nation.

61. Le Réveil d'Épiménide, suivie du Revenant, ou les préparatifs inutiles, divertissement, spectacle donné à M. et $M^{m e}$ D. à leur retour des I... Ste M..., s.l. 1788, Bibliothèque de l'Arsenal, Rf 82652 .

62. Comédie en 5 actes, d'après Hauteroche, nouvellement mise en vers libres, par M. Collé, Paris, P.-F. Gueffier fils, 1770 . 


\section{RÉSUMÉS}

Le présent article dresse le cadre d'une réflexion sur la place de la féerie et plus largement du merveilleux dans les théâtres de société de la seconde moitié du xviIIe siècle. Il existe en effet (ou il survit) une mode de ces petites pièces, qui tournent autour de fées et d'intrigues amoureuses, favorables au jeu des actrices et dont L'Oracle de Poullain de Saint-Foix est emblématique. La question est donc de savoir comment les théâtres privés, c'est-à-dire les théâtres d'éducation, de société et les fêtes privées, adoptent, reflètent ou adaptent cette mode. On trouve effectivement quelques pièces merveilleuses dans le théâtre d'éducation, les amateurs de théâtre de société jouent de temps à autre des pièces mettant en scène des personnages merveilleux et quelques fêtes sont bâties sur une "idée" qui reprend des motifs empruntés au conte. Même si ces occurrences sont loin d'être majoritaires en cette seconde moitié du siècle, elles montrent que l'esprit du merveilleux n'est pas mort sur les scènes privées bien qu'il donne rarement à voir un univers authentiquement codé. Ce sont ces modalités d'apparition et leurs valeurs particulières qui nous intéressent.

\section{Private Theatres and Fairy Tales in the Second Half of the XviII ${ }^{\text {th }}$ Century}

This article provides a framework for a reflection on the importance of fairy tales and, on a broader scale, of fantasy, in privately-owned theatres during the second half of the $\mathrm{xvIII}^{\text {th }}$ century. Indeed, the fashion for short plays focusing on fairies and love schemes existed or survived throughout the period and provided an excellent opportunity for actresses to display their theatrical skills. Poullain de Saint-Foix's L'Oracle remains the most typical example of the genre. The question at stake is to understand how private theatres, such as educational and highsociety stages, as well as private festivities, adopted, perpetuated or modified that trend. Whereas educational theatre effectively included effectively several fantasy plays, amateur actors enjoyed portraying supernatural characters on private stages, and a few special celebrations revolved around an "idea " borrowed from a fairy tale. However, while such occurrences are far from prevailing after 1750, but they show how the spirit of fantasy was kept alive on private stages, even though the universe being depicted was lacking a truly genuine code. Consequently, the major interest main point is not only to identify the conditions under which such playlets appeared, but also to highlight their intrinsic values.

\section{AUTEUR}

\section{MARIE-EMMANUELLE PLAGNOL-DIÉVAL}

Université de Paris 12 